\title{
Evolution of Fluctuation in relativistic heavy-ion collisions
}

\author{
Bedangadas Mohanty, Jan-e Alam and Tapan K. Nayak \\ Variable Energy Cyclotron Centre, Calcutta 700064, India
}

(Dated: November 10, 2018)

\begin{abstract}
We have studied the time evolution of the fluctuations in the net baryon number for different initial conditions and space time evolution scenarios. We observe that the fluctuations at the freezeout depend crucially on the equation of state (EOS) of the system and for realistic EOS the initial fluctuation is substantially dissipated at the freeze-out stage. At SPS energies the fluctuations in net baryon number at the freeze-out stage for quark gluon plasma and hadronic initial state is close to the Poissonian noise for ideal as well as for EOS obtained by including heavier hadronic degrees of freedom. For EOS obtained from the parametrization of lattice QCD results the fluctuation is larger than Poissonian noise. It is also observed that at RHIC energies the fluctuations at the freeze-out point deviates from the Poissonian noise for ideal as well as realistic equation of state, indicating presence of dynamical fluctuations.
\end{abstract}

PACS numbers: 25.75.-q,05.40.-a,12.38.Mh

\section{INTRODUCTION}

Heavy-ion collisions at relativistic energies offer a unique environment for the creation and study of the Quark-Gluon Plasma (QGP) phase of nuclear matter. In the collision process the system may go through a phase transition whereby it evolves from a very hot and dense QGP state to normal hadronic matter. A characteristic feature of this process is that the system experiences large eventby-event fluctuations in thermodynamic quantities such as temperature, number density etc. Our ability to observe a characteristic fluctuation in various observables, in present day experiments, has been facilitated by the large number of particles produced in the relativistic heavy-ion collisions at the Super Proton Synchrotron (SPS) and the Relativistic HeavyIon Collider (RHIC) along with the advent of large acceptance detectors at these experiments [1]. The near Gaussian distributions of the experimental observables like multiplicity, transverse energy [2], transverse momentum and particle ratios 3. have provided an opportunity to relate these quantities to thermodynamical properties of matter, such as specific heat [4] and matter compressibility [5]. Fluctuations as a signature of quark-hadron phase transition has been experimentally studied extensively but no definite signature has been observed in this sector so far [2, 3, 6, 7].

The main task for using fluctuation as a probe of QGP phase transition would be to identify an observable whose fluctuation will survive from the time of formation of plasma till they get detected after the freeze-out. In this context, it was suggested that the study of the fluctuation in conserved quantities e.g. net baryon number, net charge or net strangeness will be very useful $[8,9,10,11]$. The fluctuation in any conserved quantity $(\mathcal{O})$ is given by 12

$$
(\Delta \mathcal{O})^{2} \equiv\left\langle\mathcal{O}^{2}\right\rangle-\langle\mathcal{O}\rangle^{2}=T \frac{\partial\langle\mathcal{O}\rangle}{\partial \mu}
$$

where, $\mu$ is the associated chemical potential and $T$ is the temperature of the system under consideration. Here $\langle\mathcal{O}\rangle$ represents the mean value of the conserved quantity over a large number of events and $(\Delta \mathcal{O})$ denotes the deviation of its value from the mean, event-by-event. It has been shown that the fluctuation in these conserved quantities with and without QGP formation are very different. Hence, study of fluctuations in these conserved quantities can be a good signature of transition from confined to de-confined state of matter.

However, in the earlier calculations [9, 10] ideal gas EOS for both the QGP and hadronic gas was considered. For the QGP initial state, the evolution of fluctuation during mixed phase have also not been taken into account. Finally, modifications of hadronic spectral functions in the thermal bath was ignored.

The aim of this paper is to study the evolution of the fluctuation in conserved quantities from time of formation to the time of detection. In addition to the QGP and hadronic initial state we consider an important case where the hadrons are formed at the initial stage but the spectral function of the hadrons are different from their vacuum properties. We also discuss the sensitivity of the results on different EOS. Here we concentrate only on the net baryon number fluctuation and the results are presented in terms of the experimentally measured quantity, $\left(\Delta N_{b}\right)^{2} / N_{b}$, where $N_{b}$ is the number of baryons and $\Delta N_{b}$ is the fluctuation in the net baryon number.

The paper is organized as follows. In the next section we discuss the possible evolution scenarios. Then the initial conditions used for solving the evolution equations for various evolution scenarios are 
presented in section III. In section IV we study the evolution of the system. Section V and VI is devoted for the results at the freeze-out for SPS and RHIC energies respectively. And finally in section VII we present the summary and conclusions.

\section{EVOLUTION SCENARIOS}

We consider the following three possible evolution scenarios.

1. QGP scenario: The system starts evolving from an initial QGP state formed at a time $\tau_{i}$ and temperature $T_{i}$. It then expands, hence cools and reaches the critical temperature $T_{c}$ at a time $\tau_{q}$. In the mixed phase the cooling due to expansion is compensated by the heating of the system due to the liberation of the latent heat in a first order phase transition. Hence the temperature remains constant at $T_{c}$ (super-cooling is neglected here). After the mixed phase ends at a time $\tau_{h}$, further expansion takes place and finally the system disassembles at a time $\tau_{f}$ and temperature $T_{f}$. At this stage, called the freeze-out stage, the mean free path of the particles are too large to have any further interactions, and those are detected experimentally.

2. Hadron gas scenario: The hot and dense system is formed in the hadronic state at a time $\tau_{i}$ and temperature $T_{i}$ and the system expands from the initial to freeze-out state (at time $\tau_{f}$ when the temperature is $T_{f}$ ) without a phase transition.

3. Hadron gas with mass variation: The other possibility is that the system may form in the hadronic state as in (2) above, but the spectral function of the hadrons are different from their vacuum counterparts. Among others, in the present case we will consider the shift of the pole of the hadronic spectral function according to the universal scaling law proposed by Brown and Rho 13 :

$$
m_{h}^{*}=m_{h}\left(1-\frac{T^{2}}{T_{c}^{2}}\right)^{\lambda}
$$

where $m_{h}^{*}\left(m_{h}\right)$ is the in-medium (vacuum) mass of the hadrons (except pseudo-scalar) and the index $\lambda$ takes a value between 0 and 1. Here we choose $\lambda=1 / 6$ according to the well known Brown-Rho scaling [14.

\section{INITIAL CONDITIONS}

The initial conditions in terms of initial temperature $\left(T_{i}\right)$ can be set for the three scenarios from the following relation:

$$
d S=\frac{2 \pi^{4}}{45 \zeta(3)} d N=4 \frac{\pi^{2}}{90} g_{\text {eff }} T_{i}^{3} \Delta V,
$$

where $d S(d N)$ is the entropy (number) contained within a volume element $\Delta V=\pi R^{2} \tau_{i} d \eta, R$ as the radius of the colliding nuclei. $g_{\text {eff }}$ is the effective statistical degeneracy, $\zeta(3)$ denotes the Riemann zeta function and $\eta$ is the space-time rapidity. For massless bosons (fermions) the ratio of $d S$ to $d N$ is given by $2 \pi^{4} /(45 \zeta(3)) \sim 3.6(4.2)$, which is a crude approximation for heavy particles. For example the above ratio is $3.6(7.5)$ for $140 \mathrm{MeV}$ pions $(938 \mathrm{MeV}$ protons) at a temperature of $200 \mathrm{MeV}$.

First we consider the situation at SPS energies where $d N / d y \sim 700$ for $\mathrm{Pb}+\mathrm{Pb}$ collisions. For above three scenarios taking $g_{e f f}$ as given in Table $\mathbb{E}$, we obtain the initial temperature by using Eqn. 3. The values of the initial temperatures are given in Table If.

The chemical potential at the initial state is fixed by constraining the specific entropy (entropy per baryon) to the value obtained from the analysis of experimental data. The specific entropy at SPS is about 40 for $\mathrm{Pb}+\mathrm{Pb}$ collisions 15, 16, 17, 18. The net baryon number can be calculated using the baryon (anti-baryon) number density, $n_{b}\left(n_{\bar{b}}\right)$ given by,

$$
n_{b}=\frac{g}{(2 \pi)^{3}} \int f(\vec{p}) d^{3} p,
$$

where $g$ is the baryonic degeneracy. We take $g=4$ for proton and neutron. $f(\vec{p})$ is the well known Fermi-Dirac distribution,

$$
f(\vec{p})=[\exp ((E \pm \mu) / T)+1]^{-1}
$$

where $\mu(-\mu)$ is the chemical potential for baryon (anti-baryon), $E=\sqrt{p^{2}+m^{2}}$ and $n_{\bar{b}} \equiv n_{b}(\mu \rightarrow$ $-\mu)$.

For the QGP scenario we take the mass of the quarks as, $m_{q}{ }^{2}=m_{q c}{ }^{2}+m_{q t h}{ }^{2}$, where $m_{q t h}$ is the thermal mass [19] and $m_{q c}$ is the current quark mass. We have taken vacuum mass and effective mass for the hadrons (given by Eqn. 2) for cases 2 and 3 respectively. We obtain the value of the chemical potential to be $132 \mathrm{MeV}, 340 \mathrm{MeV}$ and $105 \mathrm{MeV}$ for cases (1), (2) and (3) respectively. These are also summarized in Table I.

Having fixed the initial temperature and chemical potential we now calculate the initial fluctuations 
TABLE I: Initial conditions and the initial values of fluctuation for the three scenarios.

\begin{tabular}{|lccc|}
\hline Initial Values/Scenarios & 1 & 2 & 3 \\
\hline$g_{\text {eff }}$ & 37 & 15 & 24 \\
$T_{i}(\mathrm{MeV})$ & 196 & 264 & 226 \\
$\mu_{i}(\mathrm{MeV})$ & 132 & 340 & 105 \\
$\left(\Delta N_{b}\left(\tau_{i}\right)\right)^{2} / S$ & 0.014 & 0.029 & 0.061 \\
$\left(\Delta N_{b}\left(\tau_{i}\right)\right)^{2} / N_{b, y}$ & 0.56 & 1.16 & 2.46 \\
\hline
\end{tabular}

for the three scenarios.

1. Quark Gluon Plasma: From the initial net baryon number, one can easily calculate the net baryon number fluctuations using Eqn. 1. The fluctuation in the net baryon number in the QGP phase at time $\tau_{i}$, temperature $T_{i}$ and chemical potential $\mu_{i}$, can be shown to be [10:

$$
\left(\Delta N_{b}\left(\tau_{i}\right)\right)_{\mathrm{QGP}}^{2}=\frac{2 V}{9} T_{i}^{3}\left(1+\frac{1}{3}\left(\frac{\mu_{i}}{\pi T_{i}}\right)^{2}\right),
$$

With the initial conditions as discussed above, the initial fluctuations for the QGP scenario turns out to be $\left(\Delta N_{b}\left(\tau_{i}\right)\right)_{\mathrm{QGP}}^{2}=35$. It may be noted that the value of $g$ in Eqn. 4 is taken to be 12, for two flavor case.

The entropy density in the QGP phase is calculated from Eqn. 3 with $g_{\text {eff }}=37$. The total entropy of the system is given by:

$$
S=V \frac{4 \pi^{2} g_{e f f}}{90} T_{i}^{3} \sim 2500 .
$$

For fixed initial entropy of the system the initial temperature is different for the three cases because of the different values of the $g_{\text {eff }}$. In the present work we solve the evolution equation for ideal fluid neglecting entropy generations due to various viscous effects. The total entropy is kept constant for all the three scenarios as it is obtained from the number of particles per unit rapidity measured experimentally. So the fluctuation in net baryon number per entropy is $\left(\Delta N_{b}\left(\tau_{i}\right)\right)_{\mathrm{QGP}}^{2} / S=0.014$, a value similar to that obtained in [10]. In this case the fluctuation per unit baryon, $\left(\Delta \bar{N}_{b}\left(\tau_{i}\right)\right)_{\mathrm{QGP}}^{2} / N_{b, y}$ $=0.56$ where $N_{b, y}$ is the net baryon number per unit rapidity, $d N_{b} / d y \sim 62$ for SPS energies.

2. Hadron Gas : The net baryon number fluctuation in the hadronic gas can be calculated using Eqn. 1 and is given by:

$\left(\Delta N_{b}\right)_{\mathrm{HG}}^{2}=\frac{g V}{4}\left[\frac{2 m T_{i}}{\pi}\right]^{3 / 2} \exp \left(-m / T_{i}\right) \cosh \left(\mu / T_{i}\right)$.
Substituting the values of $T_{i}, \mu_{i}$ and $m=938 \mathrm{MeV}$ for the nucleons, we obtain the fluctuation in net baryon number, $\left(\Delta N_{b}\left(\tau_{i}\right)\right)_{\mathrm{HG}}^{2} \sim 72$. The ratio of the fluctuation to total entropy is $\left(\Delta N_{b}\left(\tau_{i}\right)\right)_{\mathrm{HG}}^{2} / S=$ 0.029 . This value is also similar to the one obtained in [10]. In this case the value of $\left(\Delta N_{b}\left(\tau_{i}\right)\right)_{\mathrm{HG}}^{2} / N_{b, y}$ $=1.16$.

3. Hadron gas with mass variation in medium: The initial fluctuation for this case can be obtained using the Eqn's 4 and 5 together with Eqn. 2 used for the value of mass. The initial conditions of $T_{i}=226$ $\mathrm{MeV}$ and $\mu_{i}=105 \mathrm{MeV}$ are constrained to reproduce the hadronic multiplicity and the entropy per baryons. In this case we get $\left(\Delta N_{b}\left(\tau_{i}\right)\right)_{\mathrm{HG}, \mathrm{m}^{*}}^{2}=153$ and $\left(\Delta N_{b}\left(\tau_{i}\right)\right)_{\mathrm{HG}, \mathrm{m}^{*}}^{2} / S=0.061$. The value of $\left(\Delta N_{b}\left(\tau_{i}\right)\right)_{\mathrm{HG}, \mathrm{m}^{*}}^{2} / N_{b, y}=2.46$ here.

We observe that for the baryon number fluctuations corresponding to the above three scenarios:

$$
\frac{\left(\Delta N_{b}\left(\tau_{i}\right)\right)_{\mathrm{HG}}^{2}}{\left(\Delta N_{b}\left(\tau_{i}\right)\right)_{\mathrm{QGP}}^{2}} \sim 2 \text { and } \frac{\left(\Delta N_{b}\left(\tau_{i}\right)\right)_{\mathrm{HG}, \mathrm{m}^{*}}^{2}}{\left(\Delta N_{b}\left(\tau_{i}\right)\right)_{\mathrm{QGP}}^{2}} \sim 4
$$

This clearly shows that in the initial stage, there is a clear distinction between the three cases. The initial values of fluctuations are summarized in Table II. It will be of interest now to see, if these fluctuations (and the differences given by Eqn. 9) survive till the freeze-out. Next we discuss the evolution of this initial fluctuation for the three scenarios.

\section{EVOLUTION OF THE INITIAL FLUCTUATION}

We follow ref. 10] to study the proper time $(\tau)$ evolution of the baryon fluctuation in the space-time rapidity interval $\Delta \eta$ for different EOS as well as initial states as mentioned above. At the freeze-out point the fluctuation measured experimentally contain the residue of the initial fluctuation which survived the space time evolution and the fluctuations generated due to the exchange of baryons with the adjacent sub-volumes. We discuss each of the above cases separately in the following sub-sections.

\section{A. Dissipation of the initial fluctuation}

The difference in baryon flux $(\Delta n \bar{v})$ originating from different densities inside and outside of the subvolume $(A \tau \Delta \eta)$ leads to the following differential equation for $\Delta N_{b}(=\Delta n \bar{v} A \tau)$ :

$$
\frac{d \Delta N_{b}}{d \tau}=-\frac{\bar{v}}{2 \Delta \eta} \frac{\Delta N_{b}}{\tau}
$$




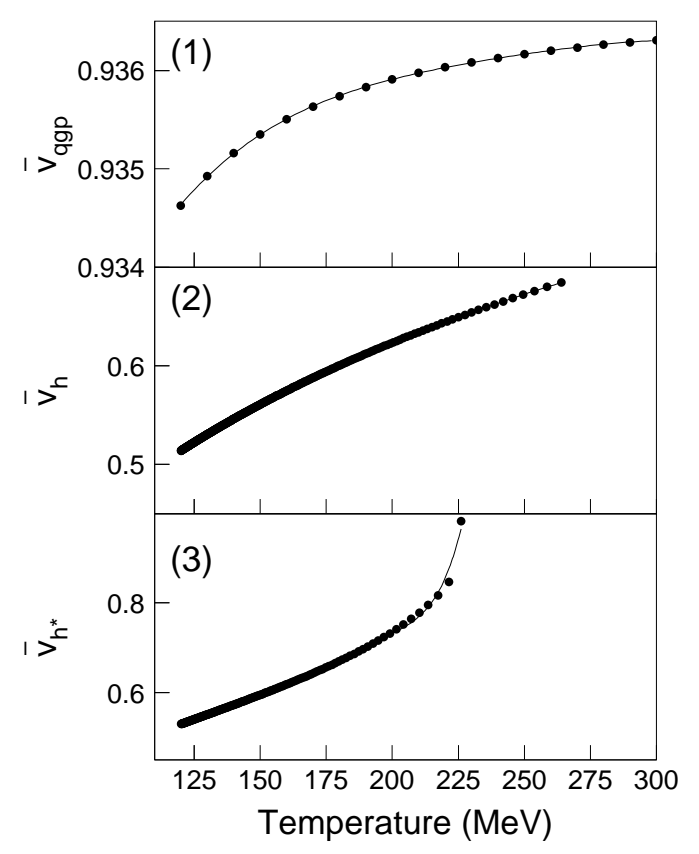

FIG. 1: Variation of average thermal velocity with temperature for three different scenarios. The results are fitted by nth-order polynomials. (1) For QGP scenario the fitting parameters are $a_{0}=0.93, a_{1}=0.11$, $a_{2}=-0.56, a_{3}=1.32$ and $a_{4}=1.20$. (2) For hadronic gas scenario with fit parameters as $a_{0}=0.19, a_{1}=4.0, a_{2}=-14.4, a_{3}=32.2$ and $a_{4}=-31.2$. (3) For hadronic gas with mass variation in medium with fit parameters as $a_{1}=-4889.8$, $a_{2}=76879, a_{3}=-0.640 E+06, a_{4}=0.297 E+07$, $a_{5}=-0.73 E+07$ and $a_{6}=0.74 E+07$.

where $\bar{v}$ is the average thermal velocity of the particles under consideration.

The solution to the differential equation 10 from initial time $\tau_{i}$ to final time $\tau_{f}$ is then given as:

$$
\Delta N_{b}\left(\tau_{f}\right)=\Delta N_{b}\left(\tau_{i}\right) \exp \left(-\frac{1}{2 \Delta \eta} \int_{\tau_{i}}^{\tau_{f}} \frac{d \tau}{\tau} \bar{v}(\tau)\right)
$$

The average thermal velocity at a given temperature can be calculated by using the following equation,

$$
\bar{v}=\frac{\int \frac{p}{E} d^{3} p f(\vec{p})}{\int d^{3} p f(\vec{p})}
$$

The values of $\bar{v}$ as a function of temperature for the three cases are shown in Fig. 11. The curves are fitted by the polynomials of the form $a_{0}+a_{1} T+$ $a_{2} T^{2}+a_{3} T^{3}+a_{4} T^{4}+a_{5} T^{5}+a_{6} T^{6}$. The values of the parameters are shown in the caption of Fig. 1. The average velocity in QGP is found to be substantially larger than the velocity in hadronic gas.

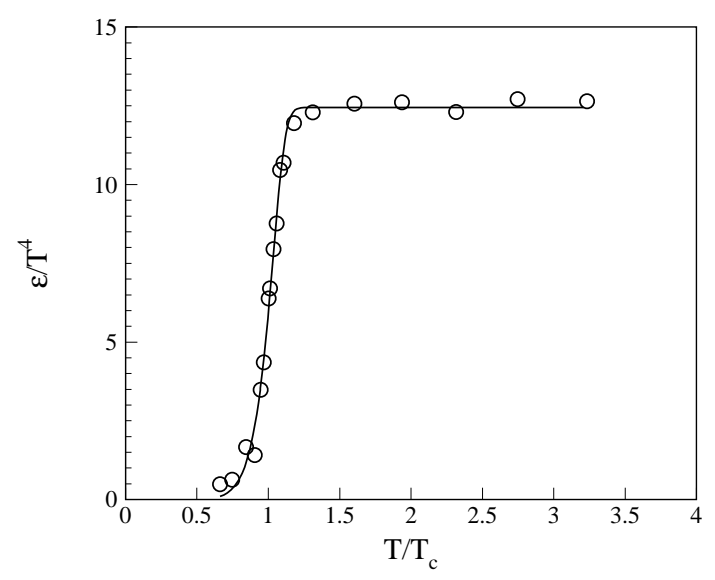

FIG. 2: The variation of energy density as a function of temperature. The result shown by solid line is obtained from Eqn. 13. Open circles show the lattice results with out the error bars.

However, in case of the hadronic system where the effective masses of the baryons (neutron and proton here) approach zero at $T_{c}$, their velocities approach the velocity of light.

The equation of state plays a vital role in deciding how the fluctuations in the net baryon number will evolve. The variation of energy density $(\epsilon)$ as a function of temperature is obtained from the 3 -flavor lattice QCD results [20] which is parametrized as follows:

$$
\epsilon=T^{4} A \tanh \left(B\left(\frac{T}{T_{c}}\right)^{C}\right),
$$

where the values of the parameters, $A, B$ and $C$ are $12.44,0.517$ and 10.04 respectively. Note that the effect of net baryons in the EOS is neglected here. Fig. 2 shows the variation of the energy density with temperature. The increase in the effective degeneracy near $T_{c}$ can be obtained from the hadronic phase with effective masses varying with temperature as in Eqn. 2. The increase in the effective degeneracy originates from the heavier hadrons going to a massless situation ( see also ref. 21, 22).

The evolution of the system under the boost invariance along the longitudinal direction is governed by the equation [23],

$$
\frac{d \epsilon}{d \tau}+\frac{\epsilon+P}{\tau}=0 ; \quad P=c_{s}^{2} \epsilon
$$

where $c_{s}$ is the velocity of the sound in the medium. To perform the integration in Eqn. 11 we need $d \tau / \tau$, which can be obtained as

$$
\frac{d \tau}{\tau}=-\left[\frac{\beta}{T}+\frac{\alpha}{\chi} \frac{d \chi}{d T}\right] d T=-f(T) d T
$$


where, $\alpha=\frac{1}{1+c_{s}^{2}}, \beta=4 \alpha$ and

$$
\chi=\left[\tanh B\left(T / T_{c}\right)^{C}\right]^{\alpha}
$$

The expression for the second term within the square bracket of Eqn.15 is given by

$$
\frac{1}{\chi} \frac{d \chi}{d T}=\alpha \frac{B C}{T}\left(\frac{T}{T_{c}}\right)^{C} \frac{1}{\cosh \left(B\left(\frac{T}{T_{c}}\right)^{C}\right) \sinh \left(B\left(\frac{T}{T_{c}}\right)^{C}\right)}
$$

Using the relation $c_{s}^{2}=\frac{S}{T} \frac{d S}{d T}=\left[3+\frac{T}{g_{\text {eff }}} \frac{d g_{\text {eff }}}{d T}\right]^{-1}$ we get the velocity of sound corresponding to the lattice QCD calculations,

$c_{s}^{2}=\left[3+B C\left(\frac{T}{T_{c}}\right)^{C} \frac{1}{\cosh \left(B\left(\frac{T}{T_{c}}\right)^{C}\right) \sinh \left(B\left(\frac{T}{T_{c}}\right)^{C}\right)}\right]^{-1}$

Now the fluctuation at the freeze-out point can be written as,

$\Delta N_{b}\left(\tau_{f}\right)=\Delta N_{b}\left(\tau_{i}\right) \exp \left(-\frac{1}{2 \Delta \eta} \int_{T_{f}}^{T_{i}} f(T) d T \bar{v}(T)\right)$

In order to study the effect of the equation of state, we will present results for three different values of $c_{s}$ : (a) $c_{s}^{2}=1 / 3$ corresponds to the ideal gas case, (b) $c_{s}^{2}=0.18$ corresponding to an EOS of hadronic gas where particles of mass upto $2.5 \mathrm{GeV}$ has been taken into account from particle data book and (c) $c_{s}^{2}$ obtained from Eqn. 18. It may be mentioned that for the ideal gas the rate of cooling is faster than the other two cases, (b) and (c).

Now we shall calculate the total dissipation of fluctuation at the freeze-out temperature. We shall consider the freeze-out temperature to be 120 $\mathrm{MeV}$ 18, 24 and a critical temperature for the QGP transition to be $170 \mathrm{MeV}$ [20]. In all the three cases below we have taken the value of $\Delta \eta=1$.

1. Quark Gluon Plasma: For the QGP initial state, the dissipation equation has three parts. In the first part, we calculate the dissipation from the initial temperature $T_{i}$ to $T_{c}$ (for the QGP phase). The second part is the dissipation during the mixed phase and in the final phase it is the dissipation from $T_{c}$ (end of mixed phase) to the freeze-out temperature $T_{f}$. Hence the complete dissipation equation becomes,

$$
\Delta N_{b}\left(T_{f}\right)=\Delta N_{b}\left(T_{i}\right) \exp \left(-\frac{1}{2 \Delta \eta}\left(\int_{T_{c}}^{T_{i}} \bar{v}_{q g p}(T) f(T) d T+\bar{v}_{m i x}\left(T_{c}\right) \ln (r)+\int_{T_{f}}^{T_{c}} \bar{v}_{h}(T) f(T) d T\right)\right)
$$

where $r$ is the ratio $\left.\left(g_{\text {eff }}\right)_{q g p} / g_{\text {eff }}\right)_{h} \sim 2.5$ and $\bar{v}_{\text {mix }} \sim \bar{v}_{q g p}\left(T_{c}\right)$.

The fluctuation in the net baryon number is evaluated for: (a) $c_{s}^{2}=1 / 3$, for which we get $\left(\Delta N_{b}\left(\tau_{f}\right)\right)_{\mathrm{QGP}}^{2} \sim 6$, (b) $c_{s}^{2}=0.18$, giving $\left(\Delta N_{b}\left(\tau_{f}\right)\right)_{\mathrm{QGP}}^{2} \sim 2.23$ and (c) $c_{s}^{2}$ as given by Eqn. 18, resulting in $\left(\Delta N_{b}\left(\tau_{f}\right)\right)_{\mathrm{QGP}}^{2} \sim 0.54$.

The initial and final fluctuation values along with the evolution process of the values for the QGP scenario is shown in Fig. 3. Only the results for $c_{s}^{2}=1 / 3$ and $c_{s}^{2}$ as given by Eqn. 18 are shown in the figures for the clarity of presentation. We have checked that the results for $c_{s}^{2}=0.18$ lies between the above two cases.

2. Hadron Gas : In case of hadronic initial state we have,

$\Delta N_{b}\left(T_{f}\right)=\Delta N_{b}\left(T_{i}\right) \exp \left(-\frac{1}{2 \Delta \eta} \int_{T_{f}}^{T_{i}} \bar{v}_{h}(T) f(T) d T\right) 21$ (a) For $c_{s}^{2}=1 / 3$, the contribution from the exponential is $\sim 0.5$. Hence the fluctuation in net baryon number is $\left(\Delta N_{b}\left(\tau_{f}\right)\right)_{\mathrm{HG}}^{2}$ is 18 , (b) for $c_{s}^{2}=0.18$, we get $\left(\Delta N_{b}\left(\tau_{f}\right)\right)_{\mathrm{HG}}^{2}$ is 14.6 and if we take (c) $c_{s}^{2}$ from Eqn. 18 the contribution from the exponential term is $\sim 0.06$, a factor of 8 smaller than the value for case (a). Consequently the fluctuation in net baryon number is $\left(\Delta N_{b}\left(\tau_{f}\right)\right)_{\mathrm{HG}}^{2}$ is 0.22 . The evolution of fluctuation for this case is depicted in Fig. 3 .

3. Hadron gas with mass variation in medium: For the hadronic initial state with mass variation,

$\left.\Delta N_{b}\left(T_{f}\right)=\Delta N_{b}\left(T_{i}\right) \exp \left(-\frac{1}{2 \Delta \eta} \int_{T_{f}}^{T_{i}} \bar{v}_{h}^{*}(T) f(T) d T\right) 22\right)$

(a) For $c_{s}^{2}=1 / 3$, the contribution from the exponential is $\sim 0.53$. Hence the fluctuation in net baryon number is $\left(\Delta N_{b}\left(\tau_{f}\right)\right)_{\mathrm{m}^{*}}^{2} \sim 44$, (b) for $c_{s}^{2}=0.18$, we get: $\left(\Delta N_{b}\left(\tau_{f}\right)\right)_{\mathrm{m}^{*}}^{2} \sim 37$ and (c) 1) taking $c_{s}^{2}$ from Eqn. 18, the contribution from the exponential term is $\sim 0.08$ a factor of 7 lower than 


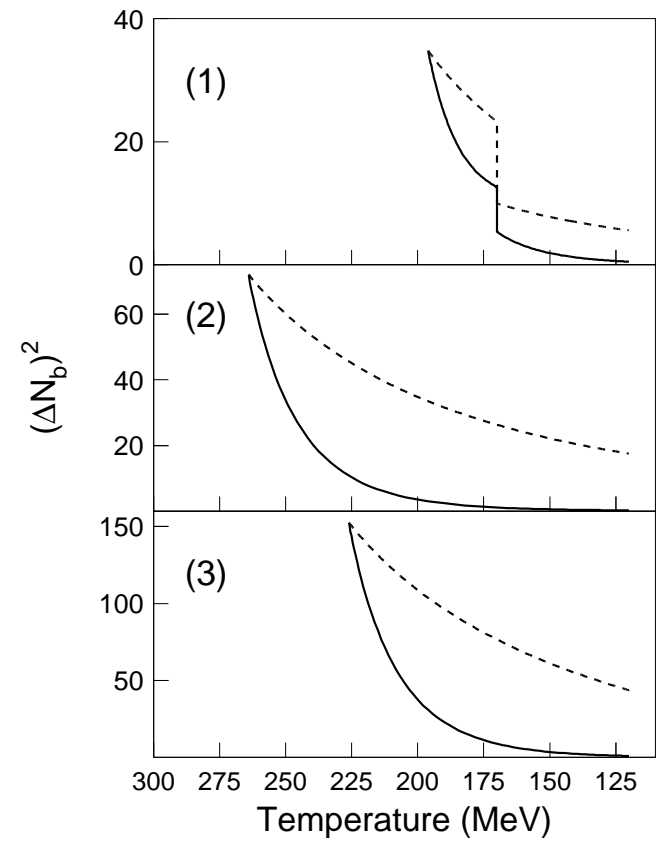

FIG. 3: The dissipation of net baryon number fluctuation for the different scenarios as a function of temperature. Note that temperature is plotted in decreasing order to reflect the evolution in time. (1) Variation of $\left(\Delta N_{b}\right)^{2}$ for QGP scenario, (2) Variation of $\left(\Delta N_{b}\right)^{2}$ for hadronic gas scenario and (3) Variation of $\left(\Delta N_{b}\right)^{2}$ for hadronic gas with mass variation scenario. The dashed lines correspond to results obtained for $c_{s}^{2}=1 / 3$, while the solid lines show the results obtained using the value of $c_{s}^{2}$ given in Eqn. 18 .

the case (a). So the fluctuation in net baryon number is $\left(\Delta N_{b}\left(\tau_{f}\right)\right)_{\mathrm{HG}, \mathrm{m}^{*}}^{2}$ is 0.92 . The evolution of fluctuation for this case is shown in Fig. 3 .

\section{B. Generation of fluctuation with time}

The baryon fluxes exchanged with neighboring sub-volumes can lead to generation of fluctuations, and is the main source that is to be detected experimentally at the freeze-out point. The total number of baryons leaving or entering $\left(N_{b}^{e x}\right)$ the sub-volume
$(A \tau \Delta \eta)$ between time $\tau_{i}$ and $\tau_{f}$ is given by 10

$$
N_{b}^{e x}\left(T_{f}\right)=\frac{N_{b}\left(T_{i}\right)}{2 \Delta \eta}\left(\int_{T_{f}}^{T_{i}} \bar{v}(T) f(T) d T\right)
$$

1. Quark Gluon Plasma: As in the previous case the fluctuations has to be evaluated in three parts in a QGP formation scenario. In the first part, we calculate the generation from the initial temperature $T_{i}$ to $T_{c}$ (for the QGP phase). The second part is the

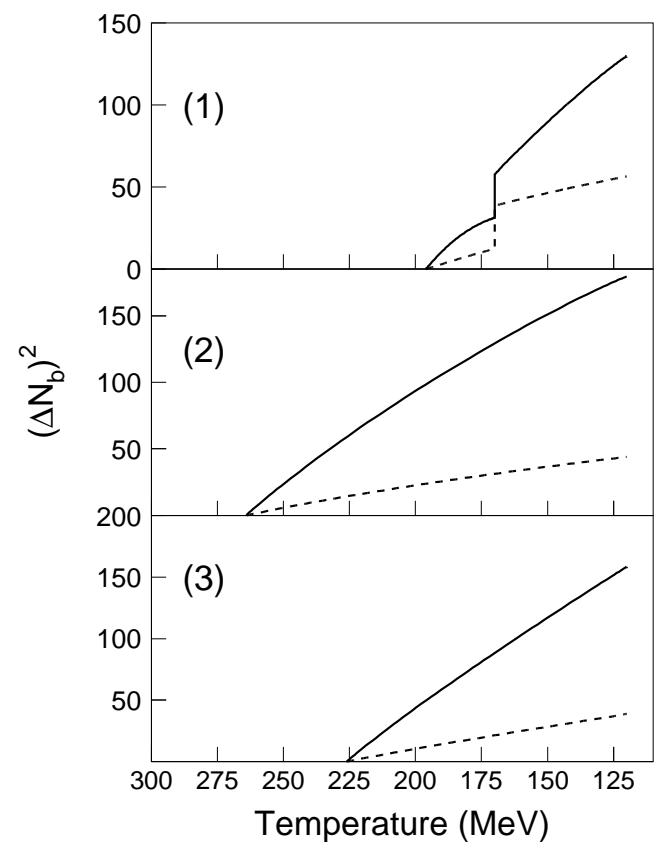

FIG. 4: The generation of net baryon number fluctuation for the different scenarios as a function of temperature. Notations are same as that of Fig. 3.

generation during the mixed phase and in the final phase it is the generation from $T_{c}$ (end of mixed phase) to the freeze-out temperature $T_{f}$. Hence the complete evolution equation becomes,

$$
\left(\Delta N_{b}\left(T_{f}\right)\right)^{2}=N_{b}\left(T_{i}\right)\left(\frac{1}{2 \Delta \eta}\left(\int_{T_{c}}^{T_{i}} \bar{v}_{q g p}(T) f(T) d T+\bar{v}_{m i x}\left(T_{c}\right) \ln (r)+\int_{T_{f}}^{T_{c}} \bar{v}_{h}(T) f(T) d T\right)\right)
$$

The fluctuation in the net baryon number is evaluated for: (a) $c_{s}^{2}=1 / 3$, for which we get
$\left(\Delta N_{b}\left(\tau_{f}\right)\right)_{\mathrm{QGP}}^{2}$ is 56.5 , (b) $c_{s}^{2}=0.18$, resulting in 
$\left(\Delta N_{b}\left(\tau_{f}\right)\right)_{\mathrm{QGP}}^{2}$ is 60.4 and (c) $c_{s}^{2}$ from Eqn. 18 giving $\left(\Delta N_{b}\left(\tau_{f}\right)\right)_{\mathrm{QGP}}^{2}$ is 129 .

The initial and final values of the fluctuations along with its time evolution for the QGP scenario are shown in Fig. 1.

2. Hadron Gas: In case of hadronic initial state we have,

$$
\left(N_{b}\left(T_{f}\right)\right)^{2}=N_{b}\left(T_{i}\right)\left(\frac{1}{2 \Delta \eta} \int_{T_{f}}^{T_{i}} \bar{v}_{h}(T) f(T) d T\right)(25)
$$

(a) For $c_{s}^{2}=1 / 3$, the fluctuation in net baryon number is $\left(\Delta N_{b}\left(\tau_{f}\right)\right)_{\mathrm{HG}}^{2}$ is 44 . (b) For $c_{s}^{2}=0.18$, we get $\left(\Delta N_{b}\left(\tau_{f}\right)\right)_{\mathrm{HG}}^{2}$ is 50 . (c) If we take $c_{s}^{2}$ from Eqn. 18, the fluctuation in net baryon number is $\left(\Delta N_{b}\left(\tau_{f}\right)\right)_{\mathrm{HG}}^{2}$ is 179 . The evolution of fluctuation for this case is displayed in Fig. 4 .

3. Hadron gas with mass variation in medium: For the hadronic initial state with mass variation,

$\left.\left(\Delta N_{b}\left(T_{f}\right)\right)^{2}=N_{b}\left(T_{i}\right)\left(\frac{1}{2 \Delta \eta} \int_{T_{f}}^{T_{i}} \bar{v}_{h}^{*}(T) f(T) d T\right) 26\right)$

(a) For $c_{s}^{2}=1 / 3$, the fluctuation in net baryon number is $\left(\Delta N_{b}\left(T_{f}\right)\right)_{\mathrm{m}^{*}}^{2} \sim 38.9$. (b) For $c_{s}^{2}=0.18$, we get $\left(\Delta N_{b}\left(T_{f}\right)\right)_{\mathrm{m}^{*}}^{2} \sim 44$. (c) Taking $c_{s}^{2}$ from Eqn. 18, the fluctuation in net baryon number is $\left(\Delta N_{b}\left(T_{f}\right)\right)_{\mathrm{HG}, \mathrm{m}^{*}}^{2}$ is 158. The evolution of fluctuation for this case is shown in Fig. 1 .

\section{FLUCTUATIONS AT THE FREEZE-OUT}

The net baryon fluctuation at the freeze-out $\left(T_{f}\right.$ $=120 \mathrm{MeV})$ is a combination of the dissipation and the generation effect as presented in the previous section. The resultant fluctuation is the sum of the variances $\left(\Delta N_{b}\left(T_{f}\right)\right)^{2}$ obtained for each of the two processes.

We present results in terms of net baryon fluctuation per unit baryon, $\left(\Delta N_{b}\left(T_{f}\right)\right)^{2} / N_{b, y}$. For Poissonian noise this value should be close to unity. Deviation from this numerical values will indicate the presence of dynamical fluctuation.

1. Quark Gluon Plasma: The final fluctuation in the net baryon number per unit baryon in the QGP scenario at the freeze-out point for the three EOS mentioned above are given by, (a) for $c_{s}^{2}=$ $1 / 3,\left(\Delta N_{b}\left(T_{f}\right)\right)_{\mathrm{QGP}}^{2} / N_{b, y} \sim 1.0$, (b) for $c_{s}^{2}=0.18$, $\left(\Delta N_{b}\left(T_{f}\right)\right)_{\mathrm{QGP}}^{2} / N_{b, y} \sim 1.0$ and (c) taking $c_{s}^{2}$ from Eqn. 18, $\left(\Delta N_{b}\left(T_{f}\right)\right)_{\mathrm{QGP}}^{2} / N_{b, y} \sim 2.0$.

2. Hadron Gas : The net baryon number fluctuation per unit baryon in the hadronic gas at freeze-out for different values of of $c_{s}$ are, (a) for
TABLE II: $\left(\Delta N_{b}\right)^{2} / N_{b, y}$ at freeze-out for three different evolution scenarios at SPS and RHIC energies.

\begin{tabular}{|clccc|}
\hline & EOS/Scenarios & 1 & 2 & 3 \\
\hline & $c_{s}^{2}=1 / 3$ & 1.0 & 1.0 & 1.3 \\
SPS & $c_{s}^{2}=0.18$ & 1.0 & 1.0 & 1.3 \\
& Lattice & 2.0 & 2.8 & 2.5 \\
& & & & \\
& $c_{s}^{2}=1 / 3$ & 1.46 & - & - \\
RHIC & $c_{s}^{2}=0.18$ & 1.96 & - & - \\
& Lattice & 3.17 & - & - \\
\hline
\end{tabular}

$c_{s}^{2}=1 / 3,\left(\Delta N_{b}\left(T_{f}\right)\right)_{\mathrm{HG}}^{2} / N_{b, y} \sim 1.0(\mathrm{~b})$ for $c_{s}^{2}=$ $0.18,\left(\Delta N_{b}\left(T_{f}\right)\right)_{\mathrm{HG}}^{2} / N_{b, y} \sim 1.0$ (c) Taking $c_{s}^{2}$ from Eqn. 18. $\left(\Delta N_{b}\left(T_{f}\right)\right)_{\mathrm{HG}}^{2} / N_{b, y} \sim 2.8$.

3. Hadron gas with mass variation in medium: The net baryon number fluctuation per unit baryon for the hadronic initial state with mass variation at freeze-out for the three EOS are, (a) for $c_{s}^{2}=1 / 3,\left(\Delta N_{b}\left(T_{f}\right)\right)_{\mathrm{HG}, \mathrm{m}^{*}}^{2} / N_{b, y} \sim 1.3$, (b) for $c_{s}=0.18,\left(\Delta N_{b}\left(T_{f}\right)\right)_{\mathrm{HG}, \mathrm{m}^{*}}^{2} / N_{b, y} \sim 1.3$ and (c) taking $c_{s}^{2}$ from Eqn. 18, $\left(\Delta N_{b}\left(\tau_{f}\right)\right)_{\mathrm{HG}, \mathrm{m}^{*}}^{2} / N_{b, y} \sim 2.5$.

The net baryon number fluctuations per unit baryon is summarized in Table II. For all the scenarios the values of the quantity $\left(\Delta N_{b}\left(\tau_{f}\right)\right)^{2} / N_{b, y}$ is larger than Poissonian noise for EOS taken from lattice QCD. This indicates presence of dynamical fluctuations. However, it should be mentioned here that the errors in lattice QCD calculations is large for temperature below the critical temperature. Furthermore $\left(\Delta N_{b}\left(\tau_{f}\right)\right)_{\mathrm{HG}}^{2} /\left(\Delta N_{b}\left(\tau_{f}\right)\right)_{\mathrm{QGP}}^{2} \sim$ 1.4 and $\left(\Delta N_{b}\left(\tau_{f}\right)\right)_{\mathrm{HG}, \mathrm{m}^{*}}^{2} /\left(\Delta N_{b}\left(\tau_{f}\right)\right)_{\mathrm{QGP}}^{2} \sim 1.25$. This means, although scenario (1) may be distinguishable from the scenarios (2) and (3) it is very difficult to differentiate (2) and (3) from fluctuation measurements. However, for EOS with $c_{s}^{2}=1 / 3$ and 0.18 they are close to Poisson value of 1 . This indicates that for these EOS; it is difficult to distinguish among the three scenarios.

\section{RESULTS AT RHIC ENERGIES}

The net baryon number fluctuation has been evaluated for RHIC energies of $\sqrt{s}=200 A \mathrm{GeV}$ of $\mathrm{Au}+\mathrm{Au}$ collisions. We have taken the total (charged and neutral) $d N / d y \sim 1100$ [25]. The initial temperatures obtained from the above multiplicity are quite large, $370 \mathrm{MeV}$ for hadronic gas and $290 \mathrm{MeV}$ 
for the case of hadronic gas with mass variation. As these temperatures are well above the critical temperature predicted by lattice QCD, we have considered only the QGP scenario for RHIC energies. We have taken the initial time to be $0.6 \mathrm{fm} / \mathrm{c}$ [26] and specific entropy to be 150 for $\mathrm{Au}+\mathrm{Au}$ collisions. For QGP we take $g_{\text {eff }}=47.5$ which gives $T_{i} \sim 251$ $\mathrm{MeV}$ and the constraint on the specific entropy gives an initial chemical potential of $73 \mathrm{MeV}$. With these initial conditions for QGP scenario at RHIC, the initial fluctuations turn out to be : $\left(\Delta N_{b}\left(\tau_{i}\right)\right)_{\mathrm{QGP}}^{2}=42$. The entropy density in the QGP phase is calculated from Eqn. 17 with $g_{\text {eff }}=47.5$ to be $\sim 3900$. So the fluctuation in net baryon number per entropy is $\left(\Delta N_{b}\left(\tau_{i}\right)\right)_{\mathrm{QGP}}^{2} / S=0.011$ and $\left(\Delta N_{b}\left(\tau_{i}\right)\right)_{\mathrm{QGP}}^{2} / N_{b, y}=$ 1.6. The value of $d N_{b} / d y \sim 26$ for RHIC energies a factor of about 2.4 lower than that for SPS energies.

As before, here the values of $T_{c}$ and $T_{f}$ are 170 and $120 \mathrm{MeV}$ respectively. (a) For $c_{s}^{2}=1 / 3$, the fluctuation in net baryon number due to dissipation at freeze-out, $\left(\Delta N_{b}\left(\tau_{f}\right)\right)_{\mathrm{QGP}}^{2}$ turns out to be 2.7. Whereas the generation mechanism gives $\left(\Delta N_{b}\left(\tau_{f}\right)\right)_{\mathrm{QGP}}^{2} \sim 35.5$. So the resultant fluctuation in net baryon number per unit baryon is $\left(\Delta N_{b}\left(\tau_{f}\right)\right)_{\mathrm{QGP}}^{2} / N_{b, y} \sim 1.46$. (b) For $c_{s}^{2}=0.18$, the fluctuation in net baryon number due to dissipation, $\left(\Delta N_{b}\left(\tau_{f}\right)\right)_{\mathrm{QGP}}^{2}$ turns out to be 2.3 . Whereas the generation mechanism gives $\left(\Delta N_{b}\left(\tau_{f}\right)\right)_{\mathrm{QGP}}^{2} \sim$ 48.7. The evolution of the dissipation and the generation of fluctuation for the QGP scenario at

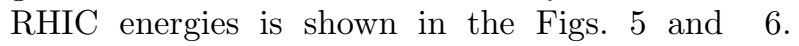
So the resultant fluctuation in net baryon number per unit baryon is $\left(\Delta N_{b}\left(\tau_{f}\right)\right)_{\mathrm{QGP}}^{2} / N_{b, y} \sim 1.96$. (c) If we take $c_{s}^{2}$ as given by Eqn. 18, the fluctuation in net baryon number due to dissipation, $\left(\Delta N_{b}\left(\tau_{f}\right)\right)_{\mathrm{QGP}}^{2}$ is 0.25 , the generation mechanism gives $\left(\Delta N_{b}\left(\tau_{f}\right)\right)_{\mathrm{QGP}}^{2} \sim 82.25$. So the resultant fluctuation in net baryon number per unit baryon is $\left(\Delta N_{b}\left(\tau_{f}\right)\right)_{\mathrm{QGP}}^{2} / N_{b, y} \sim 3.17$.

Note that though the absolute value of the fluctuations in case of RHIC is smaller than SPS the fluctuation per baryon at RHIC is larger at the freeze-out point.

\section{SUMMARY}

We have discussed the evolution of the fluctuation in net baryon number from the initial state to the final freeze-out state for three different scenarios, viz., (1) formation of QGP (2) hadronic gas and (3) hadronic gas with modified mass in the medium. In case of QGP formation we have assumed a first order phase transition. We find that the fluctuations at the initial stage agree with previously obtained values 10 where there are clear distinctions among the three cases. The fluctuations at the freeze-out point

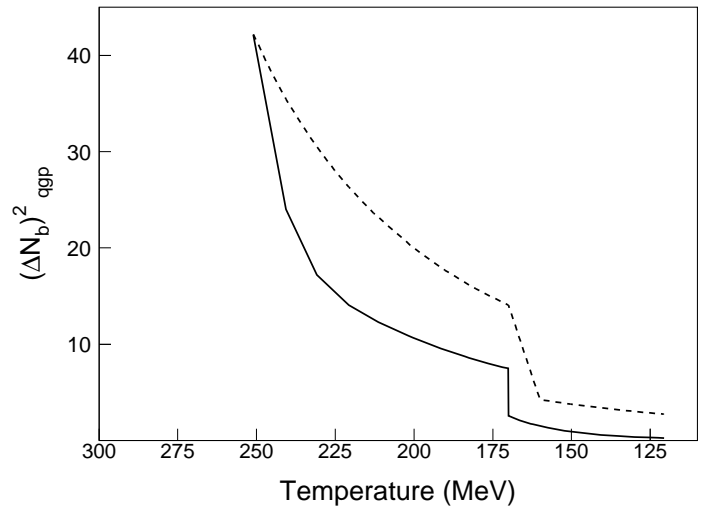

FIG. 5: The dissipation of net baryon number fluctuation for the QGP scenarios as a function of temperature calculated for $\mathrm{Au}+\mathrm{Au}$ collisions at RHIC energies. The dashed lines corresponds to results obtained for $c_{s}^{2}=1 / 3$, while the solid lines corresponds to results obtained using the value of $c_{s}^{2}$ given in Eqn. 18 .

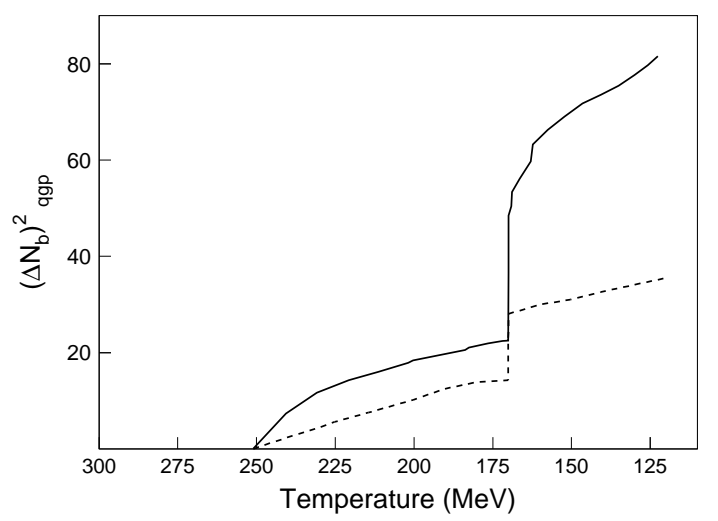

FIG. 6: The generation of net baryon number fluctuation for the QGP scenarios as a function of temperature calculated at RHIC energies of $200 \mathrm{AGev} \mathrm{Au}+\mathrm{Au}$ Collisions. Notations are same as Fig. 5 .

depend crucially on the equation of state (value of $c_{s}$ ). Fluctuations with ideal EOS is seen to dissipate at a slower rate compared to EOS from lattice calculation. At SPS energies the values of the variance depend crucially on the EOS. For EOS from lattice QCD parametrization the fluctuation at the freezeout is larger than the Poissonian noise. However, for ideal gas EOS and EOS with $c_{s}^{2}=0.18$, we do not observe fluctuations of dynamical origin. At RHIC energies the value of the fluctuations are larger than the Poissonian noise for all the three EOS under consideration here, indicating the fluctuations of dynamical origin. The effects of the finite acceptance on the fluctuation enters our calculations through 
$\Delta \eta$ and according to Eqn. 11 and 23 it is same for all the three scenarios (1), (2) and (3) discussed above. For general discussions on the effects of acceptance on the fluctuations we refer to Ref. [9, 27]. The dependence of the fluctuation on the centrality of the collisions (impact parameter) get canceled to a large extent in the ratio, $\left(\Delta N_{b}\left(\tau_{f}\right)\right)_{\mathrm{QGP}}^{2} / N_{b, y}$. It is shown in [2] that it is possible to control the impact parameter dependence of the fluctuation by measuring $E_{T}$ and analyzing data in narrow bins of $E_{T}$. A full $(3+1)$ dimensional expansion will lead to faster cooling, and hence it is interesting to see the survivability of fluctuations in such a scenario [28].

\section{Acknowledgments}

One of us (B.M.) is grateful to the Board of Research on Nuclear Science and Department of Atomic Energy, Government. of India for financial support. We would like to thank M. Asakawa for useful comments. We are thankful to the referee for his useful comments on the present manuscript.
[1] Proceedings of Quark Matter '2001, edited by T. J. Hallman, D.E. Kharzeev, J. T. Mitchell and T. Ullrich [Nucl. Phys A 698 (2002)].

[2] M.M. Aggarwal et al., (WA98 Collaboration), Phys. Rev. C65, 054912 (2002).

[3] H. Appelshauser et al., (NA49 Collaboration), Phys. Lett. B459, 679 (1999).

[4] L. Stodolsky, Phys. Rev. Lett. 75, 1044 (1995).

[5] S. Mrowczynski Phys. Lett. B430, 9 (1998).

[6] K. Adcox et. al., Phys. Rev. Lett. 89, 082301 (2002).

[7] H. Heiselberg, Phys. Rep. 351, 161 (2001).

[8] E. V. Shuryak and M. A. Stephanov, Phys. Rev. C 63, 064903 (2001).

[9] S. Jeon, V. Koch, Phys. Rev. Lett. 85, 2076 (2000).

[10] M. Asakawa, U. Heinz, B. Muller, Phys. Rev. Lett. 85, 2072 (2000).

[11] M. Prakash, R. Rapp, J. Wambach, I. Zahed, Phys. Rev. C65, 034906 (2002).

[12] L.D. Landau and E.M. Lifshitz, Statistical Physics, Part I (Pergamon, 1980).

[13] G. E. Brown and M. Rho, Phys. Rev. Lett. 66, 2720 (1991).

[14] G. E. Brown and M. Rho, Phys. Rep. 269, 333 (1996).

[15] P. Braun-Munzinger, I. Heppe and J. Stachel, Phys.
Lett. B 365, 1 (1996).

[16] J. Cleymans and K. Redlich, Phys. Rev. C60, 054908 (1999).

[17] G. Roland et. al., Nucl. Phys. A 638, 91c (1998).

[18] H. Appelshauser et.al, NA49 Collaboration, Eur. Phys. J. C2, 661 (1998).

[19] M. Le Bellac, "Thermal Field Theory", Cambridge Univ. Press, Cambridge, UK, 1996.

[20] F. Karsch, hep-ph/0103314

[21] V. Koch and G. E. Brown, Nucl. Phys. A 560, 345 (1993).

[22] G. E. Brown, H. A. Bethe, A. D. Jackson and P. M. Pizzochero, Nucl. Phys. A 560, 1035 (1993).

[23] J. D. Bjorken, Phys. Rev. D27, 140 (1983).

[24] M.M. Aggarwal et al., (WA98 Collaboration), Phys. Rev. Lett 83, 926 (1999).

[25] B.B. Back et al., (PHOBOS Collaboration), Phys. Rev. C65, 061901 (2002).

[26] A. Dumitru and D. H. Rischke, Phys. Rev. C 59 354 (1999).

[27] D. P. Mahapatra, B. Mohanty and S. C. Phatak, Int. J. Mod. Phys. A 17, 675 (2002).

[28] B. Mohanty et al., to be published. 